\title{
STRUCTURE OF SOLUTIONS TO A CHEMOTAXIS SYSTEM IN ONE SPACE DIMENSION*
}

\author{
TOSHITAKA NAGAI ${ }^{\dagger}$
}

\begin{abstract}
We consider a chemotaxis system consisting of one parabolic equation and one ordinary differential equation in one space dimension with a logarithmic chemotactic sensitivity function and the exponential growth of dynamics for the chemical attractant. The structure of positive solutions is studied from the viewpoint of infinite-dimensional dynamical systems.
\end{abstract}

Key words. chemotaxis system, structure of solutions, exact solution, invariant manifold

AMS subject classifications. 35K50, 35M10, 35B40, 92C17

1. Introduction. We consider positive solutions to the following system of differential equations

$$
\begin{array}{ll}
p_{t}=\left\{p_{x}-p(\log w)_{x}\right\}_{x} & (0<x<1, t>0), \\
w_{t}=(p-\mu) w & (0<x<1, t>0),
\end{array}
$$

where $\mu$ is a positive constant. On this system we impose no-flux boundary conditions

$$
p_{x}-p(\log w)_{x}=0 \quad(x=0,1, t>0)
$$

and initial conditions

$$
\left.p\right|_{t=0}=p_{0},\left.w\right|_{t=0}=w_{0} \quad(0<x<1) .
$$

The system is a mathematical model describing chemotaxis, that is, the directed movement of some cells in response to gradients of a chemical attractant. In the system $p(x, t)$ stands for the cell density of some species at place $x$ and time $t$, and $w(x, t)$ the concentration of a chemical attractant. Keller and Segel[13] proposed mathematical models of chemotaxis in 1970 by continuum mechanical considerations, and in 1997 Othmer and Stevens[21] derived such mathematical models of chemotaxis by using the principle of reinforced random walks. We remark that the system

$$
\left\{\begin{array}{lr}
p_{t}=D\left\{p_{x}-p(\log w)_{x}\right\}_{x} & (0<x<\ell, t>0), \\
w_{t}=\lambda p w-\mu w & (0<x<\ell, t>0) \\
p_{x}-p(\log w)_{x}=0 & (x=0, \ell, t>0) \\
\left.p\right|_{t=0}=p_{0},\left.w\right|_{t=0}=w_{0} & (0<x<\ell)
\end{array}\right.
$$

where $D, \ell, \lambda, \mu$ are positive constants, is transformed to the system (1.1)-(1.4) by the following rescaling

$$
\frac{x}{\ell} \mapsto x, \frac{D}{\ell^{2}} t \mapsto t, \frac{\ell^{2} \lambda}{D} p \mapsto p, w \mapsto w, \frac{\ell^{2} \mu}{D} \mapsto \mu .
$$

\footnotetext{
*Received June 17, 2005; accepted for publication November 30, 2005.

${ }^{\dagger}$ Department of Mathematics, Graduate School of Science, Hiroshima University, HigashiHiroshima, 739-8526, Japan (nagai@math.sci.hiroshima-u.ac.jp).
} 
We mention that there is no diffusion term present in (1.2), and this is in contrast to the usual chemotaxis systems

$$
\left\{\begin{array}{l}
u_{t}=\nabla \cdot(\nabla u-u \chi(v) \nabla v) \\
v_{t}=D \Delta v+g(u, v)
\end{array}\right.
$$

The absence of the diffusion coefficient $D$ may make differences in the structure of solutions. For example, in the case where $\chi(v)=1$ and $g(u, v)=u-v$, every positive solution under Neumann boundary conditions in one space dimension is bounded under the condition $D>0$ (see $[8,20]$ ), but the finite-time blowup of solutions may occur under the condition $D=0$ (see $[15,17]$ ). For the study of the usual chemotaxis systems, we refer to $[1,2,3,6,7,9,10,11,12,16,18,19,24,25,26]$ and references therein.

Othmer and Stevens[21] studied the system (1.1)-(1.4) numerically, and got the numerical observations indicating the possibility of finite-time blowup. Under the boundary condition $p_{x}=w_{x}=0$, Levine and Sleeman[14] constructed two kinds of exact solutions supporting the numerical observations of Othmer and Stevens. One exists globally, and another one blows up in finite time. Yang, Chen and Liu[27] also constructed the exact solutions by a different method from that in [14]. The exact solutions show that the behavior of solutions to (1.1)-(1.4) depends strongly on the initial data. For the study related to (1.1)-(1.4), we refer to $[4,15,22,23]$ and references therein.

The no-flux boundary condition (1.3) follows from the condition

$$
p_{x}(x, t)=0 \quad(x=0,1, t>0), \quad w_{0 x}(x)=0 \quad(x=0,1),
$$

because (1.2) gives the representation of $w(x, t)$

$$
w(x, t)=w_{0}(x) e^{\int_{0}^{t}\{p(x, s)-\mu\} d s},
$$

which together with the condition above implies $w_{x}(x, t)=0$ at $x=0,1$. In this paper, we study the structure of positive solutions to (1.1), (1.2) under the following boundary condition which is stronger than the boundary condition (1.3):

$$
p_{x}(x, t)=w_{x}(x, t)=0 \quad(x=0,1, t>0) .
$$

The plan of the paper is as follows. In Section 2, we discuss the behavior of positive solutions through the exact solutions given in $[14,27]$, and give another method of constructing an exact solution than that in [27]. In Section 3, we study the behavior of positive solutions from the viewpoint of infinite-dimensional dynamical systems, especially in terms of stable and unstable manifolds.

2. Exact solutions. Let $(P(x), W(x))$ be a positive solution to the stationary problem of (1.1)-(1.3):

$$
\left\{\begin{array}{l}
0=\left\{P_{x}-P(\log W)_{x}\right\}_{x}, \quad 0=(P-\mu) W \quad(0<x<1) \\
P_{x}-P(\log W)_{x}=0 \quad(x=0,1)
\end{array}\right.
$$

It is easily seen that $(P(x), W(x))$ is given by

$$
P(x) \equiv \mu, \quad W(x) \equiv \nu \quad(\nu \text { is any positive constant })
$$


For any positive constants $\alpha$ and $\beta$, the spatially homogeneous solution $(p, w)$ to $(1.1)-(1.4)$ with $p_{0}(x) \equiv \alpha, w_{0}(x) \equiv \beta$ is given by

$$
(p, w)=\left(\alpha, \beta e^{(\alpha-\mu) t}\right) .
$$

These exact solutions show that the stationary solutions $(\mu, \nu)$ are unstable, and that the large-time behavior of solutions to (1.1)-(1.4) depends on the initial data.

Concerning spatially inhomogeneous solutions, Levine and Sleeman[14] gave the following exact solutions under the boundary condition (1.5):

$$
\begin{aligned}
p(x, t) & =\pi^{2}\left[1-2 N c \varepsilon e^{N c \pi^{2} t} \frac{\varepsilon e^{N c \pi^{2} t}-\cos (N \pi x)}{1-2 \varepsilon e^{N c \pi^{2} t} \cos (N \pi x)+\varepsilon^{2} e^{2 N c \pi^{2} t}}\right], \\
w(x, t) & =e^{-\mu t} \frac{e^{\pi^{2} t}}{1-2 \varepsilon e^{N c \pi^{2} t} \cos (N \pi x)+\varepsilon^{2} e^{2 N c \pi^{2} t}},
\end{aligned}
$$

where $0<\varepsilon<1, N \in \mathbb{N}$ and $c$ is the root of $\lambda^{2}+N \lambda-1=0$. If $c$ is taken as

$$
c=\left(-N+\sqrt{N^{2}+4}\right) / 2,
$$

then

$$
\begin{aligned}
& \lim _{t \rightarrow T} p\left(x^{*}, t\right)=\lim _{t \rightarrow T} \pi^{2}\left(1+\frac{2 N c \varepsilon e^{N c \pi^{2} t}}{1-\varepsilon e^{N c \pi^{2} t}}\right)=\infty, \\
& \lim _{t \rightarrow T} w\left(x^{*}, t\right)=\lim _{t \rightarrow T} \frac{e^{-\mu t}}{1-\varepsilon e^{N c \pi^{2} t}}=\infty,
\end{aligned}
$$

where

$$
0 \leq x^{*} \leq 1, \cos \left(N \pi x^{*}\right)=1, \quad T=\frac{-1}{c N \pi^{2}} \log \varepsilon
$$

If $c$ is taken as

$$
c=\left(-N-\sqrt{N^{2}+4}\right) / 2,
$$

then the exact solution above is global in time and

$$
p(x, t) \rightarrow \pi^{2}, \quad w(x, t) e^{-\left(\pi^{2}-\mu\right) t} \rightarrow 1 \quad \text { as } t \rightarrow \infty .
$$

To construct the exact solutions in [14], they put $\psi(x, t)=\log w(x, t)+\mu t$ to transform (1.1), (1.2) with the boundary condition (1.5) into the system

$$
\left\{\begin{array}{l}
\psi_{t t}-\psi_{x x t}+\left(\psi_{x} \psi_{t}\right)_{x}=0 \quad(0<x<1, t>0), \\
\psi_{x}=0 \quad(x=0,1, t>0)
\end{array}\right.
$$

and then they seek a solution $\psi(x, t)$ in the form

$$
\psi(x, t)=t+\sum_{n=1}^{\infty} a_{n} e^{c n N t} \cos (n N \pi x)
$$

for fixed $N \in \mathbb{N}$ and $c$ real.

Yang, Chen and Liu[27] also constructed two kinds of spatially inhomogeneous exact solutions under the boundary condition (1.5). One exists globally, and another 
one blows up in finite time. They used (2.1) to construct the exact solutions by function transformations. The exact solutions are as follows:

$$
\begin{aligned}
& p(x, t)=\pi^{2}\left[\alpha-\frac{A k_{1} e^{k_{1} \pi^{2} t}+B k_{2} e^{k_{2} \pi^{2} t} \pm 2 c_{1} \sqrt{A B} e^{c_{1} \pi^{2} t} \cos (N \pi x)}{A e^{k_{1} \pi^{2} t}+B e^{k_{2} \pi^{2} t} \pm 2 \sqrt{A B} e^{c_{1} \pi^{2} t} \cos (N \pi x)}\right] \\
& w(x, t)=e^{-\mu t} \frac{e^{\alpha \pi^{2} t}}{A e^{k_{1} \pi^{2} t}+B e^{k_{2} \pi^{2} t} \pm 2 \sqrt{A B} e^{c_{1} \pi^{2} t} \cos (N \pi x)}
\end{aligned}
$$

where $\alpha>0, A>0, B>0, c_{1}<\alpha, N \in \mathbb{N}$ and

$$
k_{1}=c_{1}+N \sqrt{\alpha-c_{1}}, \quad k_{2}=c_{1}-N \sqrt{\alpha-c_{1}} .
$$

If $A>B>0, \alpha-c_{1}>N^{2}(\sqrt{A}+\sqrt{B})^{2} /(\sqrt{A}-\sqrt{B})^{2}$, then

$$
p(x, t) \rightarrow\left(\alpha-k_{1}\right) \pi^{2}(>0), \quad w(x, t) e^{-\left\{\left(\alpha-k_{1}\right) \pi^{2}-\mu\right\} t} \rightarrow \frac{1}{A} \quad(t \rightarrow \infty) .
$$

If $B>A>0, c_{1}<\alpha$, then $p(x, t)$ and $w(x, t)$ blow up at $t=T$, where

$$
T=\frac{1}{2 N \pi^{2} \sqrt{\alpha-c_{1}}} \log \frac{B}{A} .
$$

These exact solutions show that the behavior of spatially inhomogeneous solutions is sensitive to the initial data.

We give another method of constructing the exact solutions mentioned above than that in Yang, Chen and Liu[27]. To do so, for positive solutions $(p(x, t), w(x, t))$ to $(1.1),(1.2),(1.4)$ with the boundary condition $(1.5)$ we define $(\varphi(x, t), \psi(x, t))$ by

$$
\varphi(x, t)=p(x, t)-\overline{p_{0}}, \psi(x, t)=\log w(x, t)-\overline{\log w_{0}}-\left(\overline{p_{0}}-\mu\right) t .
$$

Here, $\bar{f}=\int_{0}^{1} f(x) d x$ for $f \in L^{1}(0,1)$. Then, $(\varphi(x, t), \psi(x, t))$ satisfies the system

$$
\begin{array}{ll}
\varphi_{t}-\varphi_{x x}+\overline{p_{0}} \psi_{x x}=-\left(\varphi \psi_{x}\right)_{x} & (0<x<1, t>0), \\
\psi_{t}-\varphi=0 & (0<x<1, t>0)
\end{array}
$$

with Neumann boundary conditions

$$
\varphi_{x}=\psi_{x}=0 \quad(x=0,1, t>0) .
$$

We first construct an exact solution to the system (2.5)-(2.7) in the following form

$$
\left(\begin{array}{l}
\varphi(x, t) \\
\psi(x, t)
\end{array}\right)=\sum_{n=1}^{\infty}\left(\begin{array}{l}
d_{n}(t) \\
e_{n}(t)
\end{array}\right) \chi_{n N}(x)
$$

where $d_{n}(t), e_{n}(t)$ are smooth functions, $\chi_{n}(x)=\sqrt{2} \cos \left(\sqrt{\lambda_{n}} x\right), \lambda_{n}=(n \pi)^{2}$ and $N \in \mathbb{N}$. The idea of seeking a solution in the form above comes from [15] where the existence of finite-time blowup solutions and global solutions to a chemotaxis system related to (2.5)-(2.7) are studied. We substitute (2.8) into (2.5), (2.6). Then,

$$
\partial_{t}\left(\begin{array}{c}
\varphi \\
\psi
\end{array}\right)+\left(\begin{array}{cc}
-\partial_{x}^{2} & \overline{p_{0}} \partial_{x}^{2} \\
-1 & 0
\end{array}\right)\left(\begin{array}{l}
\varphi \\
\psi
\end{array}\right)=\sum_{n=1}^{\infty}\left(\begin{array}{c}
\partial_{t} d_{n}+\lambda_{n N}\left(d_{n}-\overline{p_{0}} e_{n}\right) \\
\partial_{t} e_{n}-d_{n}
\end{array}\right) \chi_{n N}(x),
$$


and as shown in [15]

$$
\begin{aligned}
-\left(\varphi \psi_{x}\right)_{x}= & \frac{\pi^{2} N^{2}}{\sqrt{2}} \sum_{n=1}^{\infty}\left(n \sum_{m=1}^{n-1} m d_{n-m} e_{m}\right. \\
& \left.+\sum_{m=1}^{\infty} n\left\{(n+m) d_{m} e_{n+m}-m d_{n+m} e_{m}\right\}\right) \chi_{n N}(x) .
\end{aligned}
$$

Hence,

$$
\begin{aligned}
& \partial_{t} d_{n}+\lambda_{n N}\left(d_{n}-\overline{p_{0}} e_{n}\right)= \frac{\pi^{2} N^{2}}{\sqrt{2}} n \sum_{m=1}^{n-1} m d_{n-m} e_{m} \\
&+\frac{\pi^{2} N^{2}}{\sqrt{2}} \sum_{m=1}^{\infty} n\left\{(n+m) d_{m} e_{n+m}-m d_{n+m} e_{m}\right\} \\
& \partial_{t} e_{n}-d_{n}=0 .
\end{aligned}
$$

We look for $d_{n}(t), e_{n}(t)$ satisfying $e_{n}(t) \neq 0$ for all $n \geq 1$ and

$$
n d_{1} e_{n}-d_{n} e_{1}=0 \quad \text { for all } n \geq 2 .
$$

The condition (2.11) means that the term for $m=1$ in the infinite series on the right-hand side of (2.9) vanishes. Let us show that (2.11) gives the relation

$$
(n+m) d_{m} e_{n+m}-m d_{n+m} e_{m}=0 \text { for all } m, n \geq 1 .
$$

In fact, (2.11) implies

$$
\frac{d_{n}}{e_{n}}=n \frac{d_{1}}{e_{1}} \text { for all } n \geq 2 .
$$

Using this relation, we have

$$
\begin{aligned}
& (n+m) d_{m} e_{n+m}-m d_{n+m} e_{m}=e_{m} e_{n+m}\left\{(n+m) \frac{d_{m}}{e_{m}}-m \frac{d_{n+m}}{e_{n+m}}\right\} \\
& =e_{m} e_{n+m}\left\{(n+m) m \frac{d_{1}}{e_{1}}-m(n+m) \frac{d_{1}}{e_{1}}\right\} \\
& =0 .
\end{aligned}
$$

Hence, by (2.9) and (2.12), for all $n \geq 1$ the following relation holds.

$$
\partial_{t} d_{n}+\lambda_{n N}\left(d_{n}-\overline{p_{0}} e_{n}\right)=\frac{\pi^{2} N^{2}}{\sqrt{2}} n \sum_{m=1}^{n-1} m d_{n-m} e_{m} .
$$

From this relation and $(2.10)$ it follows that $e_{n}(n \geq 1)$ satisfies

$$
\partial_{t}^{2} e_{n}+\lambda_{n N} \partial_{t} e_{n}-\lambda_{n N} \overline{p_{0}} e_{n}=\frac{\pi^{2} N^{2}}{\sqrt{2}} n \sum_{m=1}^{n-1} m e_{m} \partial_{t} e_{n-m}
$$


By (2.10) and (2.11), we have $n e_{n} \partial_{t} e_{1}-e_{1} \partial_{t} e_{n}=0$ for all $n \geq 2$, from which it follows that

$$
\left|e_{n}(t)\right|=\tilde{c}_{n}\left|e_{1}(t)\right|^{n} \quad \text { for all } n \geq 1,
$$

where $\tilde{c}_{n}$ are positive constants and $\tilde{c}_{1}=1$. By (2.13) with $n=1, e_{1}(t)$ satisfies

$$
\partial_{t}^{2} e_{1}+\lambda_{N} \partial_{t} e_{1}-\lambda_{N} \overline{p_{0}} e_{1}=0
$$

which implies that

$$
e_{1}(t)=\hat{c}_{1}^{+} e^{\rho_{N}^{+} t}+\hat{c}_{1}^{-} e^{\rho_{N}^{-} t} \text { for constants } \hat{c}_{1}^{+}, \hat{c}_{1}^{-},
$$

where

$$
\rho_{N}^{ \pm}=\frac{-\lambda_{N} \pm \sqrt{\lambda_{N}^{2}+4 \overline{p_{0}} \lambda_{N}}}{2} .
$$

Assuming that $(\varphi(x, t), \psi(x, t))$ decays to zero as $t \rightarrow-\infty$, we see

$$
e_{1}(t)=\hat{c}_{1}^{+} e^{\rho_{N}^{+} t}, \quad \hat{c}_{1}^{+} \neq 0 .
$$

Hence,

$$
e_{n}(t)=c_{n} e^{n \rho_{N}^{+} t}
$$

Here, $c_{1}=\hat{c}_{1}^{+}$and $c_{n} \neq 0$ for all $n \geq 2$. To determine $c_{n}(n \geq 2)$, we substitute $e_{n}(t)=c_{n} e^{n \rho_{N}^{+} t}(n \geq 1)$ into (2.13) and get

$$
\begin{aligned}
& c_{n}\left\{\left(n \rho_{N}^{+}\right)^{2}+\lambda_{n N} n \rho_{N}^{+}-\lambda_{n N} \overline{p_{0}}\right\} e^{n \rho_{N}^{+} t} \\
& =\frac{\pi^{2} N^{2}}{\sqrt{2}} n \sum_{m=1}^{n-1} m(n-m) c_{n-m} c_{m} \rho_{N}^{+} e^{n \rho_{N}^{+} t} .
\end{aligned}
$$

Noting

$$
\left(n \rho_{N}^{+}\right)^{2}+\lambda_{n N} n \rho_{N}^{+}-\lambda_{n N} \overline{p_{0}}=N^{2}(n-1) n^{2} \pi^{2} \rho_{N}^{+},
$$

we have

$$
(n-1) n c_{n}=\frac{1}{\sqrt{2}} \sum_{m=1}^{n-1} m(n-m) c_{n-m} c_{m}
$$

Putting $n c_{n}=\sqrt{2} \varepsilon_{n}$, we have

$$
\varepsilon_{n}=\frac{1}{n-1} \sum_{m=1}^{n-1} \varepsilon_{n-m} \varepsilon_{m}
$$

From this relation, we have $\varepsilon_{n}=\varepsilon_{1}^{n}, \varepsilon_{1}=c_{1} / \sqrt{2}$, and $c_{n}=\sqrt{2} \varepsilon_{1}^{n} / n$. Therefore,

$$
e_{n}(t)=\frac{\sqrt{2}}{n} \varepsilon_{1}^{n} e^{n \rho_{N}^{+} t}, \quad d_{n}(t)=\sqrt{2} \varepsilon_{1}^{n} \rho_{N}^{+} e^{n \rho_{N}^{+} t} .
$$


For simplicity we put $\varepsilon=\varepsilon_{1}, \rho=\rho_{N}^{+}$. By the expression of $\psi(x, t)$,

$$
\psi(x, t)=\sum_{n=1}^{\infty} e_{n}(t) \chi_{n N}(x)=2 \sum_{n=1}^{\infty} \frac{1}{n} \varepsilon^{n} e^{n \rho t} \cos (n N \pi x) .
$$

Consider the case $c_{1}=\hat{c}_{1}^{+}>0$, that is, $\varepsilon>0$. Under the condition $\varepsilon e^{\rho t}<1$, we have

$$
\begin{aligned}
& 2 \sum_{n=1}^{\infty} \frac{1}{n} \varepsilon^{n} e^{n \rho t} \cos (n N \pi x)=\sum_{n=1}^{\infty} \frac{1}{n}\left\{e^{n(\log \varepsilon+\rho t+i N \pi x)}+e^{n(\log \varepsilon+\rho t-i N \pi x)}\right\} \\
& =-\log \left(1-2 \varepsilon e^{\rho t} \cos (N \pi x)+\varepsilon^{2} e^{2 \rho t}\right) .
\end{aligned}
$$

Hence,

$$
\left\{\begin{array}{l}
\psi(x, t)=-\log \left(1-2 \varepsilon e^{\rho t} \cos (N \pi x)+\varepsilon^{2} e^{2 \rho t}\right) \\
\varphi(x, t)=\partial_{t} \psi(x, t)=-2 \varepsilon \rho e^{\rho t} \frac{\varepsilon e^{\rho t}-\cos (N \pi x)}{1-2 \varepsilon e^{\rho t} \cos (N \pi x)+\varepsilon^{2} e^{2 \rho t}}
\end{array}\right.
$$

In the case $c_{1}=\hat{c}_{1}^{+}<0$, replacing $\varepsilon$ by $-\varepsilon(\varepsilon>0)$, we have an exact solution $(\varphi(x, t), \psi(x, t))$ with a plus sign of the term $\cos (N \pi x)$ in $(2.14)$.

By $p(x, t)=\overline{p_{0}}+\varphi(x, t), w(x, t)=\exp \left(\overline{\log w_{0}}+\left(\overline{p_{0}}-\mu\right) t+\psi(x, t)\right)$, we get an exact solution $(p(x, t), w(x, t))$ to $(1.1),(1.2),(1.4),(1.5)$ given by

$$
\left\{\begin{array}{l}
p(x, t)=\overline{p_{0}}-2 \varepsilon \rho e^{\rho t} \frac{\varepsilon e^{\rho t} \pm \cos (N \pi x)}{1 \pm 2 \varepsilon e^{\rho t} \cos (N \pi x)+\varepsilon^{2} e^{2 \rho t}}, \\
w(x, t)=e^{\overline{\log w_{0}}} \frac{e^{\left(\overline{p_{0}}-\mu\right) t}}{1 \pm 2 \varepsilon e^{\rho t} \cos (N \pi x)+\varepsilon^{2} e^{2 \rho t}} .
\end{array}\right.
$$

Here, $\overline{p_{0}}$ and $\overline{\log w_{0}}$ can be taken as any positive constants, $\varepsilon$ is any constant with $0<\varepsilon<1$, and $0 \leq x \leq 1, t<-(\log \varepsilon) / \rho, \rho=\rho_{N}^{+}$. To show the positivity of $p(x, t)$, we rewrite $p(x, t)$ as

$$
p(x, t)=\overline{p_{0}}-\rho+\rho \frac{1-\varepsilon^{2} e^{2 \rho t}}{1 \pm 2 \varepsilon e^{\rho t} \cos (N \pi x)+\varepsilon^{2} e^{2 \rho t}} .
$$

By noting $\varepsilon e^{\rho t}<1$ and

$$
\overline{p_{0}}-\rho=\frac{2 \overline{p_{0}}+(N \pi)^{2}-\sqrt{(N \pi)^{4}+4 \overline{p_{0}}(N \pi)^{2}}}{2}>0,
$$

the positivity of $p(x, t)$ is obtained.

We remark that the exact blowup solutions given by (2.3), (2.4) are derived from the exact solutions given by (2.15). In fact, for given $B>A>0$ and $\alpha>c_{1}$ we put

$$
\varepsilon=\sqrt{\frac{A}{B}}, \quad \overline{p_{0}}=\left\{\left(\alpha-c_{1}\right)+N \sqrt{\alpha-c_{1}}\right\} \pi^{2}, \quad \overline{\log w_{0}}=\log \frac{1}{B} .
$$

Then,

$$
\rho_{N}^{+}=N \pi^{2} \sqrt{\alpha-c_{1}}
$$


Put $k_{1}=c_{1}+N \sqrt{\alpha-c_{1}}$ and $k_{2}=c_{1}-N \sqrt{\alpha-c_{1}}$. Calculations give

$$
\begin{aligned}
\frac{1}{\pi^{2}} p(x, t) & =\left(\alpha-k_{2}\right)-\left(k_{1}-k_{2}\right) \frac{A e^{\left(k_{1}-k_{2}\right) \pi^{2} t} \pm \sqrt{A B} e^{\left(c_{1}-k_{2}\right) \pi^{2} t} \cos (N \pi x)}{B \pm 2 \sqrt{A B} e^{\left(c_{1}-k_{2}\right) \pi^{2} t} \cos (N \pi x)+A e^{\left(k_{1}-k_{2}\right) \pi^{2} t}} \\
& =\alpha-\frac{A k_{1} e^{k_{1} \pi^{2} t}+B k_{2} e^{k_{2} \pi^{2} t} \pm 2 c_{1} \sqrt{A B} e^{c_{1} \pi^{2} t} \cos (N \pi x)}{B e^{k_{2} \pi^{2} t} \pm 2 \sqrt{A B} e^{c_{1} \pi^{2} t} \cos (N \pi x)+A e^{k_{1} \pi^{2} t}}
\end{aligned}
$$

and the expression of $w(x, t)$ given by $(2.4)$.

Next, we consider the case where $(\varphi(x, t), \psi(x, t))$ decays to zero as $t \rightarrow \infty$. In this case, we see $e_{1}(t)=\hat{c}_{1}^{-} e^{\rho_{N}^{-} t}$. In the same way as the exact blowup solutions, we have the exact global solutions in time expressed by (2.15), where $\rho=\rho_{N}^{-}<0,0<\varepsilon<1$. The positivity of $p(x, t)$ holds for every $0 \leq x \leq 1, t \geq 0$ under the condition

$$
\overline{p_{0}}-\rho_{N}^{-}>(N \pi)^{2}\left(\frac{1+\varepsilon}{1-\varepsilon}\right)^{2}
$$

In fact, by $(2.16)$ with $\rho=\rho_{N}^{-}$and the relation $\overline{p_{0}}-\rho_{N}^{-}=\left(\rho_{N}^{-}\right)^{2} / \lambda_{N}$ we have

$$
\begin{aligned}
p(x, t) & \geq\left(\overline{p_{0}}-\rho_{N}^{-}\right)+\rho_{N}^{-} \frac{1+\varepsilon}{1-\varepsilon}=\left(\overline{p_{0}}-\rho_{N}^{-}\right)-N \pi \sqrt{\overline{p_{0}}-\rho_{N}^{-}} \frac{1+\varepsilon}{1-\varepsilon} \\
& =\sqrt{\overline{p_{0}}-\rho_{N}^{-}}\left(\sqrt{\overline{p_{0}}-\rho_{N}^{-}}-N \pi \frac{1+\varepsilon}{1-\varepsilon}\right)>0 .
\end{aligned}
$$

The exact solutions given by $(2.3),(2.4)$, which exist globally in time, are also derived from the exact solutions given by $(2.15)$ as follows. For given $A>B>0$ and $\alpha-c_{1}>N^{2}(\sqrt{A}+\sqrt{B})^{2} /(\sqrt{A}-\sqrt{B})^{2}$ we put

$$
\varepsilon=\sqrt{\frac{B}{A}}, \quad \overline{p_{0}}=\left\{\left(\alpha-c_{1}\right)-N \sqrt{\alpha-c_{1}}\right\} \pi^{2}, \quad \overline{\log w_{0}}=\log \frac{1}{A} .
$$

Then,

$$
\rho_{N}^{-}=-N \pi^{2} \sqrt{\alpha-c_{1}}
$$

By putting $k_{1}=c_{1}+N \sqrt{\alpha-c_{1}}$ and $k_{2}=c_{1}-N \sqrt{\alpha-c_{1}}$, calculations similar to those in the case of the exact blowup solutions give the expression of $p(x, t), w(x, t)$ given by (2.3), (2.4) respectively. We remark that the condition (2.17) is equivalent to the condition $\alpha-c_{1}>N^{2}(\sqrt{A}+\sqrt{B})^{2} /(\sqrt{A}-\sqrt{B})^{2}$, by taking into account $\overline{p_{0}}-\rho_{N}^{-}=\left(\alpha-c_{1}\right) \pi^{2}$.

Our method of constructing the exact solutions gives the reason why the way of seeking exact solutions in the form (2.2) works well, and shows that the exact solutions of [27] are derived from our exact solutions as mentioned above. It was pointed out in [15] that the exact solutions of [27] are derivable from those in [14] by translations in time or change of scale, and change of scale formulas were not given explicitly.

By the arguments above, we know that we can not construct finite-time blowup solutions and global solutions of (2.5)-(2.7), i.e., those of (1.1)-(1.3), in the form (2.8) with $d_{n}(0)=n \lambda a_{n}, e_{n}(0)=a_{n}$ except for the exact solutions above, under the condition that the sequence $\left\{\left(\hat{d}_{n}(t), \hat{e}_{n}(t)\right)\right\}=\left\{\left(n \lambda a_{n} e^{n \lambda t}, a_{n} e^{n \lambda t}\right)\right\}$ satisfies (2.13), i.e.,

$$
\partial_{t}^{2} \hat{e}_{n}+\lambda_{n N} \partial_{t} \hat{e}_{n}-\lambda_{n N} \overline{p_{0}} \hat{e}_{n}=\frac{\pi^{2} N^{2}}{\sqrt{2}} n \sum_{m=1}^{n-1} m \hat{e}_{m} \partial_{t} \hat{e}_{n-m}
$$


because $(\hat{\varphi}(x, t), \hat{\psi}(x, t))=\sum_{n=1}^{\infty}\left(\hat{d}_{n}(t), \hat{e}_{n}(t)\right) \chi_{n N}(x)$ is the exact solution of $(2.5)-$ (2.7) and $(\varphi(x, t), \psi(x, t))=(\hat{\varphi}(x, t), \hat{\psi}(x, t))$ by virtue of the condition $(2.11)$ for $\left\{\left(\hat{d}_{n}(t), \hat{e}_{n}(t)\right)\right\}$ and the uniqueness of solutions. This means that the technique used in [15] for showing the existence of finite-time blowup solutions and global solutions for a chemotaxis system related to (2.5)-(2.7) is not applicable to the existence of those to our system except for the exact solutions. In the next section we apply invariant manifold theory to studying the asymptotic behavior of solutions to (1.1), (1.2), (1.4), (1.5) as well as the existence of global solutions.

3. Stable and unstable manifolds. Throughout this section, we consider positive solutions to (1.1), (1.2), (1.4), (1.5).

We begin with mentioning the local existence as well as the uniqueness of positive solutions for the positive initial data $\left(p_{0}(x), w_{0}(x)\right)$. Consider the problem

$$
\left\{\begin{array}{l}
p_{t}=\left(p_{x}-p \psi_{x}\right)_{x}, \psi_{t}=p-\overline{p_{0}} \quad(0<x<1, t>0), \\
p_{x}=\psi_{x}=0 \quad(x=0,1, t>0), \\
\left.p\right|_{t=0}=p_{0},\left.\psi\right|_{t=0}=\log w_{0}-\overline{\log w_{0}} \quad(0<x<1) .
\end{array}\right.
$$

In what follows, as in the previous section we put

$$
\bar{f}=\int_{0}^{1} f(x) d x \quad \text { for } f \in L^{1}(0,1) .
$$

For a solution $(p(x, t), \psi(x, t))$ to $(3.1)$ satisfying $p(x, t)>0(0 \leq x \leq 1, t \geq 0)$, we get a positive solution $(p(x, t), w(x, t))$ to $(1.1),(1.2),(1.4),(1.5)$ by putting

$$
w(x, t)=e^{\overline{\log w_{0}}+\left(\overline{p_{0}}-\mu\right) t+\psi(x, t)} .
$$

The arguments of $[4,22]$ can be applied to (3.1) to getting the local existence in time and the uniqueness for classical positive solutions in a Hölder space, [17, 22] for solutions in the $L^{2}$-framework and [15] for solutions in a sequence space which is continuously and injectively imbedded in $L^{1}(0,1) \times W^{1,1}(0,1)$.

Proposition 3.1. Let $T_{m}$ be the maximal existence time of the positive solution $(p(x, t), w(x, t))$ to (1.1), (1.2), (1.4), (1.5). If $T_{m}$ is finite, then

$$
\limsup _{t \rightarrow T_{m}}\|p(t)\|_{L^{\infty}}=\infty, \quad \limsup _{t \rightarrow T_{m}}\|w(t)\|_{L^{\infty}}=\infty .
$$

This proposition means that both $p(x, t)$ and $w(x, t)$ blow up at the same time $T_{m}$. Proposition 3.1 was shown in [27] for classical solutions, and can be shown for solutions in the $L^{2}$-framework by applying the arguments of [17].

To discuss the behavior of spatially inhomogeneous positive solutions $(p(x, t), w(x, t))$, we first give the following lemma.

LEMMA 3.1. The following relation

$$
\overline{p(t)}=\overline{p_{0}}, \quad \overline{\log w(t)}=\overline{\log w_{0}}+\left(\overline{p_{0}}-\mu\right) t
$$

holds.

Proof. Integrating Eq. (1.1) on the interval $(0,1)$ in $x$ yields that

$$
\frac{d}{d t} \int_{0}^{1} p(x, t) d x=0
$$


which implies the identity on $p$.

Next, from Eq. (1.2) we have

$$
(\log w)_{t}=p-\mu .
$$

Integrating this equation on $(0,1)$ in $x$ gives the identity on $w$.

Proposition 3.2. It holds that

(i) $\|w(t)\|_{L^{1}} \geq e^{\overline{\log w_{0}}+\left(\overline{p_{0}}-\mu\right) t}$,

(ii) if $\overline{p_{0}}>\mu$, then $\lim _{t \rightarrow T_{m}}\|w(t)\|_{L^{\infty}}=\infty$.

Proof. Since the function $s \mapsto \log s$ is concave in $s>0$, Jensen's inequality gives

$$
\log \|w(t)\|_{L^{1}}=\log \int_{0}^{1} w(x, t) d x \geq \int_{0}^{1} \log w(x, t) d x=\overline{\log w(t)} .
$$

By the identity on $w$ in Lemma 3.1, this relation gives the first assertion. The second assertion follows from the first assertion in the case of $T_{m}=\infty$, and from Proposition 3.1 in the case of $T_{m}<\infty$. by

For the further study of the behavior of solutions, let us define $(\varphi(x, t), \psi(x, t))$

$$
\varphi(x, t)=p(x, t)-\overline{p_{0}}, \psi(x, t)=\log w(x, t)-\overline{\log w_{0}}-\left(\overline{p_{0}}-\mu\right) t .
$$

Then, $(\varphi, \psi)$ satisfies the system

$$
\partial_{t}\left(\begin{array}{c}
\varphi \\
\psi
\end{array}\right)+\left(\begin{array}{cc}
-\partial_{x}^{2} & \overline{p_{0}} \partial_{x}^{2} \\
-1 & 0
\end{array}\right)\left(\begin{array}{l}
\varphi \\
\psi
\end{array}\right)=\left(\begin{array}{c}
-\partial_{x}\left(\varphi \partial_{x} \psi\right) \\
0
\end{array}\right)
$$

with Neumann boundary conditions

$$
\partial_{x} \varphi=\partial_{x} \psi=0 \quad(x=0,1)
$$

and initial conditions

$$
\left.\varphi\right|_{t=0}=p_{0}-\overline{p_{0}},\left.\psi\right|_{t=0}=\log w_{0}-\overline{\log w_{0}} .
$$

We remark that the following identity

$$
\int_{0}^{1} \varphi d x=\int_{0}^{1} \psi d x=0
$$

holds by Lemma 3.1 .

Let $X$ be a Banach space defined by

$$
X=L_{0}^{2}(0,1) \times\left(H_{N}^{2}(0,1) \cap L_{0}^{2}(0,1)\right)
$$

with the norm

$$
|\Phi|_{X}=\|\varphi\|_{L^{2}}+\left\|\partial_{x}^{2} \psi\right\|_{L^{2}} \text { for } \Phi=\left(\begin{array}{c}
\varphi \\
\psi
\end{array}\right) \in X
$$


where

$$
\begin{gathered}
L_{0}^{2}(0,1)=\left\{\varphi \mid \varphi \in L^{2}(0,1), \int_{0}^{1} \varphi d x=0\right\}, \\
H_{N}^{2}(0,1)=\left\{\varphi \mid \varphi \in H^{2}(0,1), \partial_{x} \varphi=0 \text { at } x=0,1\right\} .
\end{gathered}
$$

Define a closed operator $A$ in the Banach space $X$ by

$$
A=\left(\begin{array}{cc}
-\partial_{x}^{2} & \overline{p_{0}} \partial_{x}^{2} \\
-1 & 0
\end{array}\right) \text { with the domain } D(A)=\left(H_{N}^{2} \cap L_{0}^{2}(0,1)\right)^{2}
$$

Theorem 3.1 of [17] gives the characterization of the spectrum of the following operator

$$
\left(\begin{array}{cc}
-\partial_{x}^{2} & \nu \partial_{x}^{2} \\
-1 & a
\end{array}\right) \text { for positive constants } \nu, a
$$

and this theorem is also valid for $a=0$. Applying Theorem 3.1 of [17] as $\nu=\overline{p_{0}}$ and $a=0$, we have the following theorem on the spectrum $\sigma(A)$ of the operator $A$.

THEOREM 3.1. It holds that

(i) $\sigma(A)=\left\{\lambda_{n}^{+} \mid n \geq 1\right\} \cup\left\{\lambda_{n}^{-} \mid n \geq 1\right\} \cup\left\{-\overline{p_{0}}\right\}$, where $\lambda_{n}^{ \pm}$are eigenvalues of $A$ given by

$$
\lambda_{n}^{+}=\frac{\lambda_{n}+\sqrt{\lambda_{n}^{2}+4 \overline{p_{0}} \lambda_{n}}}{2}>0, \quad \lambda_{n}^{-}=\frac{-2 \overline{p_{0}} \lambda_{n}}{\lambda_{n}+\sqrt{\lambda_{n}^{2}+4 \overline{p_{0}} \lambda_{n}}}<0,
$$

$\lambda_{n}=(n \pi)^{2}$,

(ii) $\lambda_{n}^{+} \rightarrow \infty, \quad \lambda_{n}^{-} \rightarrow-\overline{p_{0}} \quad(n \rightarrow \infty)$,

(iii) the null spaces $N\left(\lambda_{n}^{ \pm}-A\right)$ of $\lambda_{n}^{ \pm}-A$ are given by

$$
\begin{aligned}
& N\left(\lambda_{n}^{+}-A\right)=\left\{c\left(\begin{array}{c}
\lambda_{n}^{+} \\
-1
\end{array}\right) \chi_{n} \mid c \in \mathbb{C}\right\}, \\
& N\left(\lambda_{n}^{-}-A\right)=\left\{c\left(\begin{array}{c}
-\lambda_{n}^{-} \\
1
\end{array}\right) \chi_{n} \mid c \in \mathbb{C}\right\},
\end{aligned}
$$

where $\chi_{n}(x)=\sqrt{2} \cos (n \pi x)$.

The operator $A$ is sectorial in $X$. Hence, the fractional powers of $A_{1}:=A+b I$ with $\operatorname{Re} \sigma\left(A_{1}\right)>0$ are well defined, and the spaces $X^{\beta}=D\left(A_{1}^{\beta}\right)$ with the graph norm $|\Phi|_{\beta}=\left|A_{1}^{\beta} \Phi\right|_{X}$ are defined for $\beta \geq 0$. For $1 / 2<\beta<1$, the following estimate

$$
\|\varphi\|_{H^{1}}+\|\psi\|_{H^{2}} \leq \text { Const. }|\Phi|_{\beta} \quad \text { for } \Phi=\left(\begin{array}{c}
\varphi \\
\psi
\end{array}\right) \in X^{\beta}
$$

holds (see Proposition 3.2 of [17]). From this estimate it follows that the function $F$ defined by

$$
F(\Phi)=\left(\begin{array}{c}
-\partial_{x}\left(\varphi \partial_{x} \psi\right) \\
0
\end{array}\right) \quad \text { for } \Phi=\left(\begin{array}{c}
\varphi \\
\psi
\end{array}\right) \in X^{\beta} \quad\left(\frac{1}{2}<\beta<1\right)
$$

satisfies

$$
\left|F\left(\Phi_{1}\right)-F\left(\Phi_{2}\right)\right|_{\beta} \leq C\left(\left|\Phi_{1}\right|_{\beta}+\left|\Phi_{2}\right|_{\beta}\right)\left|\Phi_{1}-\Phi_{2}\right|_{\beta} \quad \text { for } \quad \Phi_{1}, \Phi_{2} \in X^{\beta},
$$


where $C$ is a constant independent of $\Phi_{1}, \Phi_{2}$. With the notation above, the system (3.3)-(3.5) can be expressed in the form

$$
\left\{\begin{array}{l}
\frac{d \Phi}{d t}(t)+A \Phi(t)=F(\Phi(t)) \quad(t>0), \\
\left.\Phi\right|_{t=0}=\Phi_{0}
\end{array}\right.
$$

where

$$
\Phi(t)=\left(\begin{array}{c}
\varphi(t) \\
\psi(t)
\end{array}\right), \quad \Phi_{0}=\left(\begin{array}{c}
p_{0}-\overline{p_{0}} \\
\log w_{0}-\overline{\log w_{0}}
\end{array}\right) .
$$

To apply invariant manifold theory to (3.7), we put

$$
\sigma(A)=\sigma_{+} \cup \sigma_{-}, \sigma_{+}=\left\{\lambda_{n}^{+} \mid n \geq 1\right\}, \sigma_{-}=\left\{\lambda_{n}^{-} \mid n \geq 1\right\} \cup\left\{-\overline{p_{0}}\right\} .
$$

Let $P_{+}$(resp. $P_{-}$) be the projection associated with $\sigma_{+}$(resp. $\sigma_{-}$), and $X_{+}=$ $P_{+} X, X_{-}=P_{-} X$. Then,

$$
\left.X=X_{+} \oplus X_{-} \quad \text { (the direct sum of } X_{+} \text {and } X_{-}\right) .
$$

We put $X_{+}^{\beta}=D\left(A_{+}^{\beta}\right)$ for the restriction of $A$ to $X_{+}$. Note that the origin $(0,0)$ in $X$ is the unique steady state of (3.7) and unstable. Applying Theorem 5.2.1 of [5] to (3.7) gives the following proposition.

Proposition 3.3. There exists $\rho>0$ such that the following holds:

(i) There exists the stable manifold $\mathcal{M}^{s}$ included in $\left\{\left.\Phi \in X^{\beta}|| \Phi\right|_{\beta} \leq \rho\right\} \quad(1 / 2<$ $\beta<1)$ which is tangent to $X_{+}^{\beta}$ at the origin. Further, if $\Phi_{0} \in \mathcal{M}^{s}$, then the solution $\Phi\left(t ; \Phi_{0}\right)$ of (3.7) with the initial function $\Phi_{0}$ exists for all $t>0$ and satisfies

$$
\left|\Phi\left(t ; \Phi_{0}\right)\right|_{\beta} \rightarrow 0 \text { exponentially as } t \rightarrow \infty .
$$

(ii) There exists the unstable manifold $\mathcal{M}^{u}$ included in $\left\{\left.\Phi \in X^{\beta}|| \Phi\right|_{\beta} \leq \rho\right\}(1 / 2<$ $\beta<1)$ which is tangent to $X_{-}$at the origin. Further, if $\Phi_{0} \in \mathcal{M}^{u}$, then the solution $\Phi\left(t ; \Phi_{0}\right)$ of (3.7) with the initial function $\Phi_{0}$ exists for all $t<0$ and satisfies

$$
\left|\Phi\left(t ; \Phi_{0}\right)\right|_{\beta} \rightarrow 0 \text { as } t \rightarrow-\infty .
$$

(iii) If $\left|\Phi\left(t ; \Phi_{0}\right)\right|_{\beta} \leq \rho$ for all $t \geq t_{0}$ (resp. $\left.t \leq t_{1}\right)$, then $\Phi\left(t ; \Phi_{0}\right) \in \mathcal{M}^{s}$ (resp. $\left.\Phi\left(t ; \Phi_{0}\right) \in \mathcal{M}^{u}\right)$ for all $t \geq t_{0}$ (resp. $\left.t \leq t_{1}\right)$.

Theorem 3.2. For the stable manifold $\mathcal{M}^{s}$ in Proposition 3.3 it holds that

(i) if $\left(p_{0}-\overline{p_{0}}, \log w_{0}-\overline{\log w_{0}}\right) \in \mathcal{M}^{s}$, then

$$
\left(p(t)-\overline{p_{0}}, \log w(t)-\overline{\log w_{0}}-\left(\overline{p_{0}}-\mu\right) t\right) \in \mathcal{M}^{s} \text { for all } t>0
$$

and

$$
\begin{gathered}
\left\|p(t)-\overline{p_{0}}\right\|_{H^{1}}+\left\|\log w(t)-\overline{\log w_{0}}-\left(\overline{p_{0}}-\mu\right) t\right\|_{H^{2}} \rightarrow 0, \\
\left\|w(t) e^{-\left(\overline{p_{0}}-\mu\right) t}-e^{\overline{\log w_{0}}}\right\|_{H^{2}} \rightarrow 0
\end{gathered}
$$

exponentially as $t \rightarrow \infty$, 
(ii) there exists $\rho_{1}>0$ such that if

$$
\left\|p(t)-\overline{p_{0}}, \log w(t)-\overline{\log w_{0}}-\left(\overline{p_{0}}-\mu\right) t\right\|_{H^{2}} \leq \rho_{1} \text { for all } t \geq t_{0},
$$

then

$$
\left(p(t)-\overline{p_{0}}, \log w(t)-\overline{\log w_{0}}-\left(\overline{p_{0}}-\mu\right) t\right) \in \mathcal{M}^{s} \text { for all } t \geq t_{0}
$$

Proof. Let $\Phi(t)={ }^{t}(\varphi(t), \psi(t))$ be the solution to (3.7) corresponding to the initial function $\Phi(0)={ }^{t}\left(p_{0}-\overline{p_{0}}, \log w_{0}-\overline{\log w_{0}}\right) \in \mathcal{M}^{s}$. Define $(p(t), w(t))$ by

$$
p(t)=\varphi(t)+\overline{p_{0}}, \quad w(t)=e^{\psi(t)+\left(\overline{p_{0}}-\mu\right) t+\overline{\log w_{0}}} .
$$

Then, $(p(t), w(t))$ is the solution to $(1.1),(1.2),(1.4),(1.5)$ corresponding to the initial function $\left(p_{0}, w_{0}\right)$, and by Proposition 3.3 ,

$$
\begin{aligned}
& (\varphi(t), \psi(t))=\left(p(t)-\overline{p_{0}}, \log w(t)-\overline{\log w_{0}}-\left(\overline{p_{0}}-\mu\right) t\right) \in \mathcal{M}^{s} \text { for all } t \geq 0, \\
& \left.\left.\right|^{t}(\varphi(t), \psi(t))\right|_{\beta} \rightarrow 0 \text { exponentially as } t \rightarrow \infty
\end{aligned}
$$

By (3.6) and (3.11), we have (3.8). Using the fact that $H^{1}(0,1) \subset C[0,1], H^{2}(0,1) \subset$ $C^{1}[0,1]$ and these injections are continuous, by (3.8) we have (3.9).

Next, let us show (ii). We note that

$$
|\Psi|_{1} \leq C\left(\left\|\phi_{1}\right\|_{H^{2}}+\left\|\phi_{2}\right\|_{H^{2}}\right) \quad \text { for } \quad \Psi=\left(\begin{array}{c}
\phi_{1} \\
\phi_{2}
\end{array}\right) \in X^{1},
$$

where $C$ is a constant independent of $\Psi$. Take $\rho_{1}>0$ such that $C \rho_{1} \leq \rho$, where $\rho$ is the one in Proposition 3.3. Then, if (3.10) is satisfied, then $|\Phi(t)|_{\beta} \leq|\Phi(t)|_{1} \leq C \rho_{1} \leq \rho$ which implies $\Phi(t) \in \mathcal{M}^{s}$ for all $t \geq t_{0}$ by Proposition 3.3.

Theorem 3.2 shows that the large-time behavior of $(p(x, t), w(x, t))$ is completely determined by $\overline{p_{0}}, \overline{\log w_{0}}$ as well as the existence of global solutions in time, under the condition $\left(p_{0}-\overline{p_{0}}, \log w_{0}-\overline{\log w_{0}}\right) \in \mathcal{M}^{s}$.

Concerning the unstable manifold, we have the following.

Theorem 3.3. For the unstable manifold $\mathcal{M}^{u}$ in Proposition 3.3 it holds that (i) if $\left(p_{0}-\overline{p_{0}}, \log w_{0}-\overline{\log w_{0}}\right) \in \mathcal{M}^{u}$, then

$$
\left(p(t)-\overline{p_{0}}, \log w(t)-\overline{\log w_{0}}-\left(\overline{p_{0}}-\mu\right) t\right) \in \mathcal{M}^{u} \text { for all } t<0
$$

and

$$
\begin{gathered}
\left\|p(t)-\overline{p_{0}}\right\|_{H^{1}}+\left\|\log w(t)-\overline{\log w_{0}}-\left(\overline{p_{0}}-\mu\right) t\right\|_{H^{2}} \rightarrow 0, \\
\left\|w(t) e^{-\left(\overline{p_{0}}-\mu\right) t}-e^{\overline{\log w_{0}}}\right\|_{H^{2}} \rightarrow 0
\end{gathered}
$$

as $t \rightarrow-\infty$,

(ii) there exists $\rho_{2}>0$ such that if

$$
\left\|p(t)-\overline{p_{0}}, \log w(t)-\overline{\log w_{0}}-\left(\overline{p_{0}}-\mu\right) t\right\|_{H^{2}} \leq \rho_{2} \text { for all } t \leq t_{1},
$$

then

$$
\left(p(t)-\overline{p_{0}}, \log w(t)-\overline{\log w_{0}}-\left(\overline{p_{0}}-\mu\right) t\right) \in \mathcal{M}^{u} \text { for all } t \leq t_{1}
$$


We next study the behavior of the positive solution $(p(x, t), w(x, t))$ as $t \rightarrow T_{m}$, where $T_{m}$ is the maximal existence time of $(p(x, t), w(x, t))$, under the condition

$$
p_{0} \neq \overline{p_{0}}, \quad\left(p_{0}-\overline{p_{0}}, \log w_{0}-\overline{\log w_{0}}\right) \in \mathcal{M}^{u} .
$$

Let $\psi(x, t)$ be the one defined by (3.2). Then, $(p(x, t), \psi(x, t))$ satisfies

$$
\left\{\begin{array}{l}
p_{t}=\left(p_{x}-p \psi_{x}\right)_{x}, \psi_{t}=p-\overline{p_{0}} \quad(0<x<1, t>0), \\
p_{x}=\psi_{x}=0 \quad(x=0,1, t>0), \\
\left.p\right|_{t=0}=p_{0},\left.\psi\right|_{t=0}=\log w_{0}-\overline{\log w_{0}} \quad(0<x<1) .
\end{array}\right.
$$

Lemma 3.2. The identity

$$
\frac{d}{d t} W(p(t), \psi(t))+Q(p(t), \psi(t))=0
$$

holds, where

$$
\begin{gathered}
W(p, \psi)=\int_{0}^{1}(p \log p-p \psi) d x \\
Q(p, \psi)=\int_{0}^{1}\left|p-\overline{p_{0}}\right|^{2} d x+4 \int_{0}^{1} e^{\psi}\left|\left(e^{-\psi / 2} \sqrt{p}\right)_{x}\right|^{2} d x .
\end{gathered}
$$

Under the condition (3.12), by Theorem 3.3 we have

$$
\lim _{t \rightarrow-\infty}\left\{\left\|p(t)-\overline{p_{0}}\right\|_{H^{1}}+\|\psi(t)\|_{H^{2}}\right\}=0 .
$$

Using Lemma 3.2 and (3.15) yields the following.

LEMMA 3.3. Under the condition (3.12), it holds that

(i) $Q(p(t), \psi(t))>0$ for every $t \in\left(-\infty, T_{m}\right)$,

(ii) the function $t \mapsto W(p(t), \psi(t))$ is decreasing in $t$ and

$$
W(p(t), \psi(t)) \rightarrow \overline{p_{0}} \log \overline{p_{0}} \quad(t \rightarrow-\infty) .
$$

Proof. Since $Q(p(t), \psi(t)) \geq 0$, by (3.14) the function $t \mapsto W(p(t), \psi(t))$ is nonincreasing. By (3.15) and $\int_{0}^{1} \psi(t) d x=0$, we have (3.16).

Suppose that $Q\left(p\left(t_{0}\right), \psi\left(t_{0}\right)\right)=0$ for some $t_{0}$. Then,

$$
p\left(t_{0}\right)=\overline{p_{0}}, \quad \int_{0}^{1}\left|\psi_{x}\left(t_{0}\right)\right|^{2} d x=0 .
$$

By $\psi\left(t_{0}\right)=$ constant and $\int_{0}^{1} \psi\left(t_{0}\right) d x=0$, we have $\psi\left(t_{0}\right)=0$. In the case of $t_{0}<0$, the uniqueness of solutions to (3.13) yields that $p(t)=\overline{p_{0}}, \psi(t)=0\left(t_{0}<t<\right.$ $\left.T_{\max }\right)$. This gives a contradiction to $p(0)=p_{0} \neq \overline{p_{0}}$. In the case of $t_{0}>0$, since $t \mapsto W(p(t), \psi(t))$ is non-increasing, combining $W\left(p\left(t_{0}\right), \psi\left(t_{0}\right)\right)=\overline{p_{0}} \log \overline{p_{0}}$ with (3.16) yields that $W(p(t), \psi(t))=\overline{p_{0}} \log \overline{p_{0}}\left(-\infty<t \leq t_{0}\right)$. Hence, we have $Q(p(t), \psi(t))=$ $0\left(-\infty<t<t_{0}\right)$, and then $p(t)=\overline{p_{0}}, \psi(t)=0\left(-\infty<t \leq t_{0}\right)$ which gives a contradiction to $p(0)=p_{0} \neq \overline{p_{0}}$. Therefore, $Q(p(t), \psi(t))>0$ for every $t<T_{m}$, and then $t \mapsto W(p(t), \psi(t))$ is decreasing by (3.14). 
THEOREM 3.4. Under the condition (3.12), the solution $(p(x, t), w(x, t))$ to (1.1), (1.2), (1.4), (1.5) with the initial function $\left(p_{0}, w_{0}\right)$ satisfies

$$
\limsup _{t \rightarrow T_{m}}\left\{\left\|p(t)-\overline{p_{0}}\right\|_{H^{1}}+\left\|w(t) e^{-\left(\overline{p_{0}}-\mu\right) t}-e^{\overline{\log w_{0}}}\right\|_{L^{\infty}}\right\}=\infty .
$$

Proof. If $T_{\max }<\infty$, then (3.17) holds by Proposition 3.1. Hence, we consider only the case of $T_{m}=\infty$. To show (3.17) with $T_{m}=\infty$, by $w e^{-\left(\overline{p_{0}}-\mu\right) t}=e^{\overline{\log w_{0}}+\psi}$ it suffices to show that

$$
\limsup _{t \rightarrow \infty}\left\{\|p(t)\|_{H^{1}}+\left\|\psi^{+}(t)\right\|_{L^{\infty}}\right\}=\infty
$$

where $\psi^{+}=\max \{\psi, 0\}$.

Assume that (3.18) is not true. Then,

$$
\sup _{t \geq 0}\left\{\|p(t)\|_{H^{1}}+\left\|\psi^{+}(t)\right\|_{L^{\infty}}\right\}<\infty .
$$

Integrating (3.14) in time $t$ and using (3.16), we have

$$
\int_{-\infty}^{t} Q(p(s), \psi(s)) d s=-W(p(t), \psi(t))+\overline{p_{0}} \log \overline{p_{0}}
$$

Noting that

$$
\sup _{t \geq 0} \int_{0}^{1} p \psi d x \leq \sup _{t \geq 0} \int_{0}^{1} p \psi^{+} d x<\infty,
$$

we see that $\sup _{t \geq 0}(-W(p(t), \psi(t)))<\infty$ by $(3.19)$, and that $\int_{-\infty}^{\infty} Q(p(s), \psi(s)) d s$ is finite by (3.20). Hence,

$$
\int_{-\infty}^{\infty}\left\|p(s)-\overline{p_{0}}\right\|_{L^{2}}^{2} d s<\infty
$$

Since $\sup _{t \geq 0}\|p(t)\|_{H^{1}}<\infty$ and $H^{1}(0,1)$ is compactly imbedded in $C[0,1]$, there exists $\left\{t_{n}\right\}$ such that

$$
t_{n} \rightarrow \infty, \quad\left\|p\left(t_{n}\right)-\overline{p_{0}}\right\|_{C[0,1]} \rightarrow 0 \quad(n \rightarrow \infty) .
$$

By $\int_{0}^{1} \psi(t) d x=0$ and (3.19), we note that

$$
\sup _{t \geq 0} \int_{0}^{1}|\psi(t)| d x=2 \sup _{t \geq 0} \int_{0}^{1} \psi^{+}(t) d x<\infty .
$$

Using (3.21), we then have

$$
\begin{aligned}
& \int_{0}^{1} p\left(x, t_{n}\right) \psi\left(x, t_{n}\right) d x \\
& =\int_{0}^{1}\left\{p\left(x, t_{n}\right) \psi\left(x, t_{n}\right)-\overline{p_{0}} \psi\left(x, t_{n}\right)\right\} d x \rightarrow 0 \quad(n \rightarrow \infty) .
\end{aligned}
$$


Combining this with (3.21) implies

$$
W\left(p\left(t_{n}\right), \psi\left(t_{n}\right)\right) \rightarrow \overline{p_{0}} \log \overline{p_{0}} \quad(n \rightarrow \infty) .
$$

Hence, by (3.20) we have

$$
\int_{-\infty}^{\infty} Q(p(s), \psi(s)) d s=\lim _{n \rightarrow \infty} \int_{-\infty}^{t_{n}} Q(p(s), \psi(s)) d s=0 .
$$

This is a contradiction to $\int_{-\infty}^{\infty} Q(p(s), \psi(s)) d s>0$. $\square$

We mention a relation between the exact solutions $(p(x, t), w(x, t))$ given by $(2.3)$, (2.4) and the invariant manifolds $\mathcal{M}^{s}, \mathcal{M}^{u}$ mentioned above.

Consider the first case where $A>B>0, \alpha-c_{1}>N^{2}(\sqrt{A}+\sqrt{B})^{2} /(\sqrt{A}-\sqrt{B})^{2}$. Then, $(p(x, t), w(x, t))$ satisfies

$$
p(x, t) \rightarrow\left(\alpha-k_{1}\right) \pi^{2}(>0), w(x, t) e^{-\left\{\left(\alpha-k_{1}\right) \pi^{2}-\mu\right\} t} \rightarrow \frac{1}{A} \quad(t \rightarrow \infty) .
$$

Put $p_{0}(x)=p(x, 0)$ and $w_{0}(x)=w(x, 0)$. We show that

$$
\begin{aligned}
\overline{p_{0}} & =\left(\alpha-k_{1}\right) \pi^{2}, \\
\overline{\log w_{0}} & =\log \frac{1}{A} .
\end{aligned}
$$

To show (3.22), we use Lemma 3.1 and $\lim _{t \rightarrow \infty} p(x, t)=\left(\alpha-k_{1}\right) \pi^{2}$ and get

$$
\overline{p_{0}}=\lim _{t \rightarrow \infty} \int_{0}^{1} p(x, t) d x=\left(\alpha-k_{1}\right) \pi^{2} .
$$

To show (3.23), we rewrite $w_{0}(x)$ in the form

$$
w_{0}(x)=\frac{1}{A\left(1+\varepsilon^{2} \pm 2 \varepsilon \cos (N \pi x)\right)}, \quad \varepsilon=\sqrt{\frac{B}{A}}<1
$$

and get

$$
\int_{0}^{1} \log w_{0}(x) d x=\log \frac{1}{A}-\int_{0}^{1} \log \left(1+\varepsilon^{2} \pm 2 \varepsilon \cos (N \pi x)\right) d x .
$$

Since

$$
\int_{0}^{1} \log \left(1+\varepsilon^{2} \pm 2 \varepsilon \cos (N \pi x)\right) d x=0
$$

we obtain (3.23).

Next, by taking into account (3.22) and (3.23), it follows from the expression of the exact solution $(p(x, t), w(x, t))$ that

$$
\begin{aligned}
& p(x, t)-\overline{p_{0}}=\pi^{2} \frac{B\left(k_{1}-k_{2}\right) e^{-\left(k_{1}-k_{2}\right) \pi^{2} t} \pm 2\left(k_{1}-c_{1}\right) \sqrt{A B} e^{-\left(k_{1}-c_{1}\right) \pi^{2} t} \cos (N \pi x)}{A+B e^{-\left(k_{1}-k_{2}\right) \pi^{2} t} \pm 2 \sqrt{A B} e^{-\left(k_{1}-c_{1}\right) \pi^{2} t} \cos (N \pi x)}, \\
& \log w(x, t)-\overline{\log w_{0}}-\left(\overline{p_{0}}-\mu\right) t \\
& =\log \frac{A}{A+B e^{-\left(k_{1}-k_{2}\right) \pi^{2} t} \pm 2 \sqrt{A B} e^{-\left(k_{1}-c_{1}\right) \pi^{2} t} \cos (N \pi x)}
\end{aligned}
$$


By $k_{1}-k_{2}=2 N \sqrt{\alpha-c_{1}}$ and $k_{1}-c_{1}=N \sqrt{\alpha-c_{1}}$,

$$
\left.\| p(t)-\overline{p_{0}}, \log w(t)-\overline{\log w_{0}}-\left(\overline{p_{0}}-\mu\right) t\right) \|_{H^{2}} \rightarrow 0 \quad(t \rightarrow \infty) .
$$

Hence, Theorem 3.2 implies that

$$
\left(p(t)-\overline{p_{0}}, \log w(t)-\overline{\log w_{0}}-\left(\overline{p_{0}}-\mu\right) t\right) \in \mathcal{M}^{s} \text { for all } t>>1 .
$$

Consider the second case where $B>A>0, c_{1}<\alpha$. We also see that

$$
\overline{p_{0}}=\left(\alpha-k_{2}\right) \pi^{2}, \quad \overline{\log w_{0}}=\log \frac{1}{B} .
$$

Since

$$
\left.\| p(t)-\overline{p_{0}}, \log w(t)-\overline{\log w_{0}}-\left(\overline{p_{0}}-\mu\right) t\right) \|_{H^{2}} \rightarrow 0 \quad(t \rightarrow-\infty),
$$

Theorem 3.3 implies that

$$
\left(p(t)-\overline{p_{0}}, \log w(t)-\overline{\log w_{0}}-\left(\overline{p_{0}}-\mu\right) t\right) \in \mathcal{M}^{u} \text { for all } t<<-1 .
$$

\section{REFERENCES}

[1] P. BILER, Local and global solvability of some parabolic systems modelling chemotaxis, Adv. Math. Sci. Appl., 8 (1998), pp. 715-743.

[2] S. Childress and J. K. Percus, Nonlinear aspects of chemotaxis, Math. Biosci., 56 (1981), pp. 217-237.

[3] H. Gajewski And K. Zacharias, Global behavior of a reaction-diffusion system modelling chemotaxis, Math. Nachr., 195 (1998), pp. 77-114.

[4] A. Friedman and J. Ignacio Tello, Stability of solutions of chemotaxis equations in reinforced random walks, J. Math. Anal. Appl., 272 (2002), pp. 138-163.

[5] D. Henry, Geometric Theory of Semilinear Parabolic Equations, Lecture Note in Math., 840, Springer, 1981.

[6] M. A. Herrero and J. J. L. Velázquez, Chemotaxis collapse for the Keller-Segel model, J. Math. Biol., 35 (1996), pp. 177-194.

[7] M. A. Herrero and J. J. L. Velázquez, A blow-up mechanism for a chemotactic model, Ann. Scuola Normale Sup. Pisa, XXIV (1997), pp. 633-683.

[8] T. Hillen and A. Potapov, The one-dimensional chemotaxis model: global existence and asymptotic profile, Math. Meth. Appl. Sci., 27 (2004), pp. 1783-1801.

[9] D. Horstmann, From 1970 until present: The Keller-Segel model in chemotaxis and its consequences I, Jahresber. Deutsch. Math.-Verein., 105:3 (2003), pp. 103-165.

[10] D. Horstmann, From 1970 until present: The Keller-Segel model in chemotaxis and its consequences II, Jahresber. Deutsch. Math.-Verein., 106:2 (2004), pp. 51-69.

[11] D. Horstmann and M. Winkler, Boundedness vs. blow-up in a chemotaxis system, J. Differential Equations, 215 (2005), pp. 52-107.

[12] W. JäGER AND S. LuCKhaus, On explosions of solutions to a system of partial differential equations modelling chemotaxis, Trans. Amer. Math. Soc., 329 (1992), pp. 819-824.

[13] E. F. Keller And L. A. Segel, Initiation of slime mold aggregation viewed as an instability, J. Theor. Biol., 26 (1970), pp. 399-415.

[14] H. A. Levine And B. D. Sleeman, A system of reaction diffusion equations arising in the theory of reinforced random walks, SIAM J. Appl. Math., 57 (1997), pp. 683-730.

[15] H. A. Levine AND J. Rencewowicz, Singularity formation in chemotaxis-a conjecture of Nagai, SIAM J. Appl. Math., 65 (2004), pp. 336-360.

[16] T. NAGAI, Blow-up of radially symmetric solutions to a chemotaxis system, Adv. Math. Sci. Appl., 5 (1995), pp. 581-601.

[17] T. NAGAi AND T. NAKAKI, Stability of constant steady states and existence of unbounded solutions in time to a reaction-diffusion equation modelling chemotaxis, Nonlinear Anal., 58 (2004), pp. 657-681. 
[18] T. Nagai, T. Senba And T. Suzuki, Chemotactic collapse in a parabolic system of mathematical biology, Hiroshima Math. J., 30 (2000), pp. 463-497.

[19] V. NAnjundiah, Chemotaxis, signal relaying, and aggregation morphology, J. Theor. Biol., 42 (1973), pp. 63-105.

[20] K. Osaki AND A. YAGI, Finite dimensional attractor for one-dimensional Keller-Segel equations, Funkcialaj Ekvacioj, 44 (2001), pp. 441-469.

[21] H. G. Othmer And A. Stevens, Aggregation, blowup, and collapse: The ABC's of taxis in reinforced random walks, SIAM J. Appl. Math., 57 (1997), pp. 1044-1081.

[22] M. RASCle, Sur un équation intégro-différentielle non linéaire issue de la biologie, J. Differential Equations, 32 (1979), pp. 420-453.

[23] M. Rascle And C. Ziti, Finite time blow-up in some models of chemotaxis, J. Mathe. Biol., 33 (1995), pp. 388-414.

[24] R. SchaAf, Stationary solutions of chemotaxis system, Trans. Amer. Math., 292 (1985), pp. 531-556.

[25] T. Senba And T. Suzuki, Chemotactic collapse in a parabolic-elliptic system of mathematical biology, Adv. Differential Equations, 6 (2001), pp. 21-50.

[26] A. YAGI, Norm behavior of solutions to the parabolic system of chemotaxis, Math. Japonica, 45 (1997), pp. 241-265.

[27] Y. Yang, H. Chen And W. LiU, On existence of global solutions and blow-up to a system of reaction-diffusion equations modelling chemotaxis, SIAM J. Math. Anal., 33 (2001), pp. $763-785$. 